\title{
Construction des connaissances, politique de santé en périnatalité et état de santé périnatale
}

\author{
Building Knowledge, Perinatal Public Health Policy and Perinatal Health Outcomes
}

\author{
A. Serfaty - G. Bréart \\ (C) Lavoisier SAS 2014
}

« Savoir ce qui précède et ce qui suit, vous aidera à mieux comprendre ce qui se passe. "

\section{Canto LXXVII}

Ezra Pound, Los cantos

Toute action individuelle ou collective, en matière de politique publique et notamment en matière de politique sanitaire implique une première étape de mobilisation des connaissances permettant de caractériser le problème à traiter. Ainsi, dans le domaine de la périnatalité, pour identifier les actions de prévention ou de prise en charge à mettre en œuvre, qu'elles soient à l'échelle d'un territoire, d'une région ou d'un pays, la construction des connaissances est une étape indispensable.

Cette construction évalue l'état de santé périnatale, les besoins à couvrir et repère les facteurs de risques et les différents déterminants susceptibles d'expliquer un décalage entre un état de santé observé et un état de santé souhaité, voire souhaitable [1,2].

Pour ce faire, elle utilise l'ensemble des données disponibles, émanant de champs scientifiques différents : médecine, épidémiologie, démographie et plus généralement les sciences sociales et politiques, et intègre de plus l'expression des usagers ou des associations qui les représentent.

Parler d'état de santé périnatale, d'indicateurs, de système (s) d'information, impose de définir les termes «périnatalité », « santé périnatale », « indicateurs ».

\footnotetext{
A. Serfaty $(\bowtie)$

AP-HP, hôpitaux universitaires est parisien, département de l'information médicale, Armand Trousseau, La Roche-Guyon, Rothschild, Paris, France e-mail : annie.serfaty@trs.aphp.fr

\section{A. Serfaty $\cdot$ G. Bréart} Inserm UMR 1153,

équipe de recherche en épidémiologie obstétricale, périnatale et pédiatrique (EPOPé), Paris, France
}

\author{
De la « période périnatale », définie à des fins \\ statistiques à la " périnatalité », couvrant \\ un champ beaucoup plus vaste : \\ de la conception à la petite enfance
}

Le terme « périnatalité » appartient à la langue française depuis 1970, et se rapporte à la période périnatale ; adjectif utilisé dans la langue française depuis 1952 [3]. Lors de l'Assemblée mondiale de la santé, l'Organisation mondiale de la santé (OMS) adopte la définition de la « période périnatale », comme étant « la période qui commence 22 semaines (154 jours) après le début de gestation (c'est-à-dire au moment où le poids de naissance est normalement de 500 grammes) et se termine sept jours révolus après la naissance " [4]. Cette définition à visée statistique et épidémiologique s'étend dans la pratique à une période beaucoup plus vaste. En effet, tel que le définit le dictionnaire des termes de médecine Garnier-Delamarre, le champ de la périnatalité couvre l'ensemble des conditions et des soins qui entourent la grossesse, l'accouchement, la naissance et le suivi du nouveau-né dans ses premiers mois de vie [5].

Les textes relatifs aux Commissions Régionales de la Naissance (CRN) de 1999 intègrent ces différents temps. Ils sont concernés par les aspects du soin et de la prévention, notamment la prévention des troubles de la relation mèreenfant, ainsi que les aspects relevant du handicap à la naissance, l'accueil de l'enfant et l'accompagnement de l'entourage. La circulaire relative aux CRN promeut les conditions de sécurité pendant la grossesse et l'accouchement, une meilleure information et responsabilisation des femmes et des couples, par la préparation à la naissance et les aspects de régulation des naissances (contraception et interruption volontaire de grossesse) [6]. Le temps autour de la conception, nommé « périconceptologie » par le Professeur Uzan lors de l'inauguration du bâtiment Salat-Baroux (hôpital Trousseau, Paris) le 11 octobre 2007, en fait partie [7].

La périnatalité recouvre donc un champ relativement étendu, allant de l'avant conception, période qui concerne

1. Résolution WHA20.19 et WHA43.24 
les femmes en âge de procréer, jusqu'à la petite enfance, voire jusqu'à l'âge de six ans pour les réseaux en santé périnatale, puisque l'on s'intéresse aux conséquences de la " période périnatale ». La périnatalité englobe des périodes déterminantes pour le développement du capital santé à l'âge adulte et trouve sa traduction dans l'appellation et les missions de la Commission nationale de la naissance et de la santé de l'enfant (CNNSE) installée en 2011.

\section{État de santé périnatale : des sources de données disparates, des indicateurs}

L'état de santé périnatale n'est pas facile à établir, que ce soit dans la population générale, dans celles des femmes en âge de procréer ou encore sur des groupes de population. Nous ne disposons pas d'indicateur global pour pouvoir mesurer ce que serait un «état de santé périnatale » et le comparer aux pays semblables à la France.

La mesure de l'état de santé se construit à partir d'indicateurs de nature diverse, notamment : des indicateurs sociodémographiques, des indicateurs de mortalité, des indicateurs de morbidité pour mesurer soit l'incidence, soit la prévalence d'une pathologie, des indicateurs de risque pour estimer un nombre de personnes exposées ou une probabilité avec laquelle une pathologie donnée peut se déclencher, des indicateurs d'utilisation des services de santé, des indicateurs de consommation de soins ou de prévention, des indicateurs de ressources ou encore des indicateurs de connaissance, de pratiques, de comportements ou de croyances sur la santé périnatale de la part des usagers ou des professionnels de la reproduction, de la grossesse, de l'accouchement, de la naissance et de l'enfance $[1,2]$.

Renseigner les indicateurs de santé périnatale nécessite tout d'abord de recenser l'existant, donc :

- D'identifier les sources de recueil, à savoir :

- des recueils de routine et institutionnels, notamment : l'état civil pour les événements naissances, les décès néonatals, les décès infantiles, le Programme de médicalisation des système d'information (PMSI) pour les séjours hospitaliers, les consultations, actes et examens complémentaires en médecine de ville ou hospitalière, les certificats de santé de l'enfant, les déclarations d'IVG, les interruptions médicales de grossesse (IMG), les naissances par procréation médicalement assistée (PMA), le dépistage combiné de la trisomie 21 , etc. ;

- des recueils faisant appel à des enquêtes régulières, des cohortes ou ad hoc, notamment l'Enquête nationale périnatale (ENP) ; l'Enquête nationale confidentielle sur les morts maternelles (ENCMM) ; les Registres du handicap, les registres de malformations, les registres de mortinatalité, l'Étude épidémiologique sur les petits âges gestationnels (EPIPAGE), l'étude «Épidémiologie de la morbidité maternelle sévère » (EPIMOMS), l'Étude Longitudinale Française depuis l'Enfance (ELFE), etc. ;

- de repérer les sources de données, notamment : le PMSI, le Système national d'information inter-régimes de l'assurance maladie (SNIIRAM), l'INSEE, la Statistique annuelle des établissements (SAE), l'Association des utilisateurs de dossiers informatisés en pédiatrie, obstétrique et gynécologie (Audipog), le Baromètre santé, l'ENP, l'ENCMM, l'Agence de biomédecine (ABM), le CEPIDc, l'enquête FECOND, l'enquête KABP, l'ENNS, l'enquête nationale ESTEBAN, l'enquête INCA ;

- de mobiliser les organismes producteurs d'indicateurs, notamment : l'INSEE, l'INSERM (CépiDc...), la DRESS, l'INED, la CNAMTS, l'INVS.

Pour répondre aux impératifs de suivi des cent objectifs de la loi relative à la politique de santé publique du 9 août 2004, des rapports sur l'état de santé des français sont élaborés, documentant ainsi les indicateurs associés aux objectifs de la loi. Depuis, six rapports annuels ont vu le jour, ceux de 2006, 2007, 2008, 2009-2010, 2011 et celui de 2014, dans la préfiguration de la future loi de santé publique [8].

Les objectifs de la loi relative à la politique de santé publique du 9 août 2004, qui concernent la santé reproductive et la périnatalité sont au moins cinq, à savoir : 1) le taux de mortalité maternelle ${ }^{2}$;2) le taux de mortalité périnatale ${ }^{3}$; 3) le taux de complications des grossesses extra-utérines responsables d'infertilité ; 4) la fréquence des situations périnatales à l'origine de handicaps à long terme ; 5) l'accès à une contraception adaptée, à la contraception d'urgence et à l'IVG dans de bonnes conditions pour toutes les femmes qui décident d'y avoir recours. Il en existe d'autres de nature

2. Dans la classification internationale des maladies (CIM-10) de l'OMS, la mort maternelle se définit comme « le décès d'une femme survenu au cours de la grossesse ou dans un délai de 42 jours après sa terminaison, quelle qu'en soit la durée ou la localisation, pour une cause quelconque déterminée ou aggravée par la grossesse ou les soins qu'elle a motivés, mais ni accidentelle ni fortuite ». Les morts maternelles se subdivisent en deux groupes. Les décès par cause obstétricale directe : ce sont « ceux qui résultent de complications obstétricales (grossesse, travail et suites de couches), d'interventions, d'omissions, d'un traitement incorrect ou d'un enchaînement d'événements résultant de l'un quelconque des facteurs ci-dessus ». Le deuxième groupe correspond aux décès par cause obstétricale indirecte : ce sont « ceux qui résultent d'une maladie préexistante ou d'une affection apparue au cours de la grossesse sans qu'elle soit due à des causes obstétricales directes, mais qui a été aggravée par les effets physiologiques de la grossesse ».

3. Le taux de mortalité périnatale est défini comme le nombre d'enfants naissant sans vie ou décédés à moins de sept jours pour 1000 naissances (enfants nés vivants ou sans vie). Cet indicateur était produit chaque année par l'INSEE à partir de l'état civil. 
transversale, comme la consommation d'alcool, de tabac ou encore les comportements alimentaires... [9]

Quant au projet EUROPERISTAT, il a pour objectif d'établir un système de surveillance de la santé périnatale en Europe pour 26 pays membres de l'Union Européenne, plus l'Islande, la Norvège et la Suisse, à partir d'une liste d'indicateurs communs. Elle est constituée de dix indicateurs essentiels $^{4}$, de 20 indicateurs recommandés et des indicateurs en cours de développement. Ils sont classés selon quatre groupes : 1) la santé périnatale ; 2) la santé maternelle ; 3) les caractéristiques de la population et les facteurs de risques et 4 ) le système de soins [10]. Cette liste d'indicateurs a été retenue pour l'évaluation des réseaux de santé périnatale dans la circulaire de 2006 [11].

Pour les indicateurs, les taux de mortalité maternelle et périnatale, retenus pour le suivi du plan gouvernemental périnatalité (2005-2007) «Humanité, proximité, sécurité, qualité », les données disponibles permettent d'établir quelques repères sur l'état de santé périnatale [12].

Le taux de mortalité maternelle est estimé à 9,6 décès maternels pour 100000 naissances vivantes sur la période 2007-2009, ce qui ne présente pas d'augmentation par rapport à celui de la période 2004-2006, alors même que certains facteurs de risque restent présents comme l'augmentation de l'âge maternel à l'accouchement. Il tend à stagner depuis le milieu des années 2000. Ce taux reste d'un niveau comparable à celui observé en GrandeBretagne ou aux Pays-Bas. Le dernier rapport du CNEMM publié en 2013 estime qu'en moyenne 85 décès maternels surviennent par an, avec un taux d'évitabilité supérieur à $50 \%$. Les causes principales restent l'hémorragie du postpartum et l'hypertension artérielle. Il est rapporté des inégalités dans l'excès de décès chez les femmes d'Afrique subsaharienne ou selon les régions, notamment en Île de France $[13,14]$.

Le taux de mortalité périnatale, construit pour mesurer les décès en lien avec la période périnatale, est reparti à la hausse depuis 2000. Il est passé de 36,0 pour 1000 naissances en 1950 à 6,6 décès pour 1000 naissances en 2000, pour augmenter après 2002 à un taux supérieur à 10,0 pour 1000 naissances jusqu'au milieu des années 2000. En 2007, le taux de mortalité périnatale s'établit à 10,9 pour 1000 naissances (enfants nés vivants ou sans vie). Le taux de mortinatalité suit une évolution similaire à celle du taux

4. Les dix indicateurs essentiels sont : E1 : taux de mortinatalité ; E2 : taux de mortalité néonatale ; E3 : taux de mortalité infantile ; E4 : distribution du poids de naissance; E5 : distribution de l'âge gestationnel ; E6 : taux de mortalité maternelle (selon l'âge et le mode d'accouchement) ; E7 : taux de grossesses multiples ; E8 : distribution de l'âge maternel ; E9 : distribution de la parité ; E10 : distribution des naissances selon le mode d'accouchement (selon la parité, le nombre de fœtus, la présentation fœtale et un antécédent de césarienne). de la mortalité périnatale : il est estimé à 9,3 pour 1000 enfants nés vivants ou sans vie en 2007, 9,2 pour 1000 en 2010 (ENP). Si les changements de réglementation intervenus en 2001 et en 2008 peuvent expliquer une partie de la hausse de cet indicateur, ils n'expliquent probablement pas la totalité de cette évolution [15].

D'autres indicateurs sont renseignés, comme le taux de mortalité néonatale ${ }^{5}$ et de mortalité infantile ${ }^{6}$ qui ont arrêté de baisser depuis 2005. Le taux de mortalité néonatale est estimé à 2,3 pour 1000 naissances vivantes en 2010 (2,6\% en 2003). Le taux de mortalité infantile se stabilise autour de 3,8 pour 1000 naissances vivantes. Des disparités territoriales sensibles, particulièrement entre la métropole et les départements d'outremer, persistent pour l'ensemble de ces indicateurs [8].

Le taux de prématurité 7 est estimé à $6,6 \%$ en 2010 à partir des données de l'ENP et 6,9\% en 2010 avec le PMSI ; taux en augmentation par rapport aux données de l'ENP 2003 $(6,3 \%)$; place la France au $10^{\mathrm{e}}$ rang après l'Islande, l'Irlande. Cette proportion est beaucoup plus élevée pour les naissances multiples $(41,7 \%)$. L'augmentation de la grande prématurité peut être expliquée notamment par : l'augmentation des grossesses multiples, l'augmentation de l'âge à la maternité, les traitements de la stérilité et les fécondations in vitro, les décisions de provoquer l'accouchement à des petits âges gestationnels pour éviter au nouveau-né une souffrance in utero.

La proportion d'enfant de moins de 2500 grammes est estimée en 2010 à $6,4 \%$ [15].

PREMUP estime à $20 \%$, la proportion des grossesses pathologiques, mettant en jeu la santé de la mère et/ou de l'enfant ; ceci est principalement lié à la prématurité et au retard de croissance de l'enfant (RCIU). Un handicap sur deux de l'enfant à la naissance proviendrait de ces grossesses pathologiques [https://premup.org/fra/accueil].

La mise en commun des différentes données permettent d'appréhender l'état de santé périnatale. Cependant, le calcul des indicateurs ne portent pas toujours sur les mêmes périodes, voire sur les mêmes critères, ou encore sur les mêmes unités d'analyse (événement selon le lieu de survenue ; événement selon le lieu du domicile). Les données utilisées pour construire certains indicateurs ne sont pas toujours validées, ou encore leur recueil ne s'inscrit pas dans une démarche d'assurance qualité.

\footnotetext{
5. Taux de mortalité néonatale : rapport du nombre annuel de décès néonataux (enfants nés vivants décédés avant 28 jours) à l'ensemble des naissances vivantes.

6. Taux de mortalité infantile : rapport du nombre annuel de décès d'enfants nés vivants décédés avant un an à l'ensemble des naissances vivantes.

7. Naissances survenant avant 37 semaines d'âge gestationnel révolu.
} 
Un contexte démographique : des naissances légèrement moins nombreuses en 2013 $(810000)$, un indicateur conjoncturel de fécondité $\left(\mathrm{ICF}^{8}\right)$ qui tend à baisser

En France, chaque année, plus d'un million de femmes débutent une grossesse.

Environ 15000 femmes font une grossesse extra-utérine, 220000 ont une interruption volontaire de grossesse, 120000 à 130000 font une fausse couche spontanée et plus de 800000 femmes donnent naissance à un nouveau-né, dont près de 23500 suite à une méthode de procréation médicalement assistée, soit environ une naissance sur 40 ; quatre fois plus qu'il y a 20 ans.

Le nombre de naissances demeure supérieur à celui des trente dernières années. Il était de 775441 en 1983 ; stable jusque fin des années 1990 pour atteindre un pic en 2000 avec 807405 naissances. Il diminue jusqu'en 2004 pour augmenter à nouveau en 2005 avec 806822 naissances et atteindre un pic en 2010 de 832799 naissances. En 2013, 810000 bébés sont nés dans la France entière (hors Mayotte) soit près de 11000 bébés en moins qu'en 2012 (821 000 naissances). Comme l'explique l'Institut national de la statistique et des études économiques (INSEE), le niveau de naissances résulte du nombre de femmes en âge de procréer qui diminue depuis 1995 et de la fécondité qui commence à décroître légèrement : l'Indice conjoncturel de fécondité étant passé de 2,03 en 2010, à 2,01 en 2012 puis 1,99 en 2013. Par ailleurs, l'âge moyen des mères à l'accouchement atteint 30,1 ans en 2013 , soit 0,6 an de plus qu'il y a dix années [16].

La statistique annuelle des établissements de santé (SAE) recense près de 552 centres périnatals, dont 9 de proximité en France en 2012, où sont recensées 816991 naissances dont 6997 mort-nés pour la même année (SAE). En 2004, ils étaient 684 centres périnatals dont 84 de proximité, recensant 791762 naissances, dont 5927 mort-nés (SAE).

\section{La santé périnatale, un des domaines prioritaires de l'action publique}

La santé périnatale a été l'un des domaines prioritaires de la politique de santé publique ayant donné lieu à de nombreux plans et programmes, depuis les années 1940 avec, notamment, la création de la protection maternelle et infantile.

\footnotetext{
8. Est la somme des taux de fécondité par âge observés sur une année donnée. Cet indicateur donne le nombre moyen d'enfants qu'aurait une femme tout au long de sa vie si les taux de fécondité observés à chaque âge l'année considérée demeuraient inchangés. Il est parfois exprimé en nombre d'enfants pour 100 femmes.
}

Les années 1970 ont vu la politique nationale périnatale se développer autour d'une organisation centrée sur l'offre de soins hospitalière pour s'orienter au cours du temps sur la gestion du risque médicopsychosocial, formalisé autour de la mise en place des réseaux de santé dès la fin des années 1990. Un élément structurant de la politique de santé périnatale a été la « régionalisation des soins périnatals » avec les décrets dits de sécurité périnatale de 1998. Elle s'appuie sur la gradation des soins, élément fondateur de l'orientation des femmes vers le site de naissance adapté aux facteurs de risque de la mère et du futur nouveau-né ; enjeu majeur pour la qualité et la sécurité des soins périnatals. Cet axe est introduit dans le Plan gouvernemental (1995-2000) et a fait l'objet de recommandations de bonnes pratiques [17].

Le concept de sécurité à la naissance a progressivement dépassé la sécurité physique pour s'élargir au concept de sécurité psychosociale et affective dans sa dimension environnementale. Il a pris sa place dans le Plan périnatalité (2005-2007) « Humanité, Proximité, Sécurité, Qualité » et dans le cadre des réseaux en santé périnatale [18].

La future loi de santé publique prévue pour 2015 a pour ambition de replacer l'usager dans un parcours de santé qui soit conforme à ses besoins, à son profil, à ses difficultés et ainsi renforcer la gradation des soins et l'approche territoriale.

\section{État de santé périnatale : un système d'information à construire}

L'objectif du dossier intitulé "état de santé périnatale : quels indicateurs? quel(s) système(s) d'information? » est de contribuer à répondre aux questions sous-jacentes : comment mesure-t-on l'état de santé périnatale en France ? Comment peut-on identifier les facteurs explicatifs d'une situation périnatale souhaitée mais non atteinte, afin de mieux identifier les leviers d'action?

Cette réflexion s'impose devant l'absence d'amélioration significative des indicateurs de santé périnatale.

Nous savons que de nombreuses sources existent. Nous savons aussi que les données ne sont pas toutes disponibles et accessibles, validées et fiables. Nous savons qu'il y a une volonté de rendre disponibles les données sur l'état de santé de la population avec les rapports de suivi des objectifs de la politique de santé publique.

En effet, la construction des indicateurs périnatals font appel aux nombreuses sources de recueil et de données disparates, pas toujours interopérables ${ }^{9}$ entre elles. Or, pour

9. L'interopérabilité est la capacité qu'ont plusieurs systèmes d'échanger de l'information entre eux et d'utiliser l'information qui a été échangée [ASIP Santé, cadre d'interopérabilité des systèmes d'information de santé (CI-SIS) 
caractériser l'état de santé périnatale, il est nécessaire de pouvoir renseigner les indicateurs pour les différents états et étapes périnatales, en prenant en compte la dimension de la femme, de l'enfant et de son entourage. Pour mieux identifier les facteurs de risque, la discontinuité dans le parcours périnatal, la communicabilité des données entre elles et leur chainage seront nécessaires pour mieux comprendre et mieux agir.

Compte tenu de la multitude de sources de recueils et de données disparates, non interopérables, nous ne pouvons considérer qu'il existe à ce jour un système d'information en périnatalité.

Le constat avait été posé en 1999 dans le livre blanc sur le Réseau national d'information sur la naissance : créer un "système d'information " spécifique à notre spécialité et un "réseau national d'information sur la naissance " est une nécessité en raison de l'insuffisance et de l'incohérence des moyens institutionnels d'information en périnatalité " [19]. Le Plan gouvernemental (2005-2007) propose : «Le partage des données médicales permettrait de disposer d'indicateurs à même de mieux fonder les décisions en matière de prise en charge médicale et d'organisation de l'offre de soins.... » [12]. Le rapport de la Cour des comptes de 2012 pointe une dégradation du système d'information [20].

Dans le cadre de ce dossier, il est apparu important de rappeler les étapes des réponses des pouvoirs publics, pour mieux comprendre la genèse des politiques et leur mise en œuvre. Un article de synthèse intitulé « L'histoire des réponses des pouvoirs publics dans le domaine de la santé périnatale en France (1945-2014) » est consacré aux différents plans gouvernementaux.

L'article intitulé «Les réseaux de santé en périnatalité : mise en place d'indicateurs communs, une place pour le suivi de l'état de santé périnatale (résultats en 2012) 》 propose d'illustrer comment, à partir d'indicateurs définis, les informations recueillies à un niveau territorial, renseignées par chaque réseau, vont être en capacité ou non de calculer des indicateurs nationaux, en passant par une validation intra- et inter-réseau.

L'article intitulé « Suivi des indicateurs de pratiques cliniques en périnatalité : l'exemple des hémorragies sévères du post-partum à partir de la base de données du réseau Sentinelle Audipog (1994-2009) » propose d'illustrer, à partir d'une base de données impliquant des maternités volontaires (AUDIPOG), l'analyse notamment d'une pratique lors de l'accouchement, " la délivrance dirigée », dans le cadre de la prévention des hémorragies du post-partum (HPP), une des causes de mortalité maternelle et un indicateur de qualité et de sécurité des soins.

L'article intitulé « Les données sur les maternités à disposition des usagers. Quelles données? Pour quoi faire?» vient éclairer la demande d'information par les usagers de la santé. Cet article fait l'état des lieux des différentes bases de données existantes, des informations disponibles et de leur accessibilité. L'accès à l'information santé est indispensable dans le parcours de santé périnatal de la femme enceinte. C'est par l'accès à cette information que la femme enceinte et son entourage deviennent acteur de leur parcours coordonné de soins.

Une mise au point sur la construction de l'indicateur de mortinatalité et ses difficultés, dans un contexte où les modalités d'enregistrement à l'état civil ont été modifiées en août 2008 sera proposé dans un texte intitulé : «Le cas des mort-n és : le taux de mortinatalité, un indicateur difficile à mesurer » dans le prochain numéro de mars 2015.

Ce dossier vient s'inscrire dans l'actualité de la préparation de la future loi relative à la politique de santé publique. Devant l'absence d'amélioration significative des indicateurs de santé périnatale, face à une politique de santé active dans le domaine de la périnatalité, la question d'un système d'information réactif, qui permette une veille sur les indicateurs, permettrait à terme d'identifier les facteurs sur lesquels il serait urgent d'ajuster les interventions nécessaires à l'amélioration des résultats en termes de santé périnatale.

Ce dossier n'a pas la prétention d'épuiser le sujet tant la dimension de la santé périnatale est un champ vaste et qui traverse la vie de toute personne mais aussi de nombreux champs de la santé publique. En effet, c'est la construction des indicateurs, notamment de résultats de santé, voire de connaissances qu'ont les acteurs et les usagers sur la santé périnatale, qui permettra de mieux préciser l'action individuelle et/ou collective à mettre en place pour atteindre des indicateurs en santé périnatale plus performants. La place des réseaux en santé périnatale, des bonnes pratiques professionnelles, ou encore celle de l'usager est indispensable pour qu'un système d'information raisonnée puisse être construit [21].

\section{Références}

1. Brownson RC, Baker EA, Leet TL, et al (2003) Evidence-based public health. New York : Oxford University press

2. Pineault R, Daveluy C (2000) La planification de la santé. Concepts, méthodes stratégies. In: La planification de la santé. Concepts, méthodes stratégies. R. Pineault and C. Daveluy, Editors. Agence d'Arc Inc. (Les Editions) : Montréal. p. 480

3. Rey A (2005) Dictionnaire culturel en langue française. Le Robert Paris. p. 1568

4. World Health Organization (2004) ICD-10 International statistical classification of diseases and related health problems: tenth revision. 2nd ed, ed. WHO. Vol. 2. Geneva. 131

5. Garnier, Delamare (2012) Dictionnaire illustré des termes de médecine. Maloine, Paris, p. 1054

6. Direction générale de la santé (1999) Circulaire DGS/SP nº9$394 \mathrm{du} 6$ juillet 1999 relative à la mise en place des commissions régionales de la naissance. Bulletin officiel Paris. p. 3

7. Uzan S (2007) Périconceptologie. Paris 
8. DRESS, DGS (2014) Indicateurs de suivi de l'état de santé de la population Révision 2013 - Rapport Final, série sources et Méthodes Eds p. 524

9. Loi n²004-806 du 9 août 2004 relative à la politique de santé publique. (2004) Journal Officiel de la République Française ${ }^{\circ}$ 185 du 11 août

10. Euro-Peristat project with SCPE and Eurocat (2013) European Perinatal Health Report; the health of pregnant women and babies in Europe 2010. www.europeristat.com

11. DHOS, Union Nationale des Caisses d'Assurance Maladie (2006) Circulaire $\mathrm{N}^{\circ} \mathrm{DHOS} / \mathrm{O} 1 / \mathrm{O} 3 / \mathrm{CNAMTS} / 2006 / 151 \mathrm{du}$ 30 mars 2006 relative au cahier des charges national des réseaux de santé en périnatalité. Ministère de la Santé et des Solidarités, Assurance maladie des salariés, Sécurité sociale Caisse nationale p. 20

12. Ministère de la Santé et de la Protection Sociale (2004) Plan « périnatalité » 2005-2007 : humanité, proximité, sécurité, qualité. Ministère de la Santé et de la Protection Sociale: Paris. p. 44

13. Saucedo M, Deneux-Tharaux C, Bouvier-Colle MH, et al (2013) Ten years of confidential inquiries into maternal deaths in France, 1998-2007. Obstet Gynecol 122:752-60
14. Saucedo M, Deneux-Tharaux C, Bouvier-Colle MH (2013) [Maternal mortality in France, 2007-2009]. J Gynecol Obstet Biol Reprod (Paris). 42:613-27

15. Blondel B, Lelong N, Kermarrec M, Goffinet F (2012) Trends in perinatal health in France from 1995 to 2010. Results from the French National Perinatal Surveys. J Gynecol Obstet Biol Reprod (Paris) 41:e1-e15

16. Bellamy V, Beaumel C (2014) Bilan démographique 2013. Trois mariages pour deux Pacs. I. Première, Editor. 2014, Insee. p. 4

17. Ministère des Affaires sociales de la santé et de la ville (1994) Pour un nouveau plan périnatalité. Ministère chargé de la santé : Paris, p. 44

18. Molénat F (2004) Périnatalité et prévention en santé mentale. Collaboration médico-psychologique en périnatalité. DHOS, Editor Paris. p. 26

19. Maria B, Mamelle N (1999) France-PERINAT. Réseau national d'information sur la naissance. Un projet réaliste pour le troisième millénaire. CNGOF Audipog. p. 34

20. Cour des comptes (2012) La politique de périnatalité : l'urgence d'une remobilisation. Cour des comptes p. 34

21. Serfaty A, Gold F, Benifla JL, Breart G (2011) From knowledge to planning considerations: a matrix to assess health needs for the perinatal network in eastern Paris. Eur J Public Health 21:504-11 